(C) 2012 IEEE. Personal use of this material is permitted. Permission from IEEE must be obtained for all other uses, in any current or future media, including reprinting/republishing this material for advertising or promotional purposes, creating new collective works, for resale or redistribution to servers or lists, or reuse of any copyrighted component of this work in other works. 


\title{
Characterization of Ultra Non Linear SOA in a heterodyne detector configuration with remote Photonic Local Oscillator distribution
}

\author{
A. R. Criado, Student Member, IEEE, C. de Dios and P. Acedo, Member, IEEE
}

\begin{abstract}
A heterodyne receiver scheme based on electrooptical (EO) downconversion is introduced. The local oscillator (LO) and the retrieved intermediate frequency (IF) signals are remotely distributed over fiber to/from a single Ultra Non Linear semiconductor optical amplifier device (SOA) that acts as a highly nonlinear mixer device, enhancing the downconversion performance of the system. The photonic LO is generated modulating a DFB under Gain Switching (GS) eliminating the need of external elements and the polarization dependency. We evaluate the performance of the downconversion up to frequencies much higher than the bandwidth of the devices (15 GHz). Results show broadband heterodyne detection performance of the system. The presented scheme offers advantages in terms of compactness, cost efficiency and can be easily scaled to remotely reach multiple receivers in scalable detection arrays.
\end{abstract}

Index Terms-Microwave photonics, heterodyne receiver, optical mixing, Semiconductor Optical Amplifier,

\section{INTRODUCTION}

M ICROWAVE photonics has allowed to overcome many of the limitations related to traditional electronic systems in terms of bandwidth, electromagnetic interference (EMI) immunity and power losses, becoming a fundamental technology for signal distribution, broadband communications and optical signal processing [1, 2]. Among the variety of new technologies that have recently arisen following this approach [2], the study of photonic heterodyne receivers in the $\mathrm{GHz}$ range is an important field of study, for which the development of an optoelectronic mixer would be advantageous, allowing direct downconversion of the received RF signal to an intermediate frequency (IF) modulated on an optical carrier for further distribution and processing. Such component and their associated functionality could be directly integrated in the photonic local oscillator distribution architectures that are common place today in Radar and other fields [3].

Several optical mixing schemes have been already

Manuscript received February 01, 2012. Work supported by the Spanish Ministry of Science and Technology through the project TEC2009-14525-C0202. The work by A.R. Criado has been supported by the Spanish Ministry of Science and Technology under the FPI Program, Grant\# BES2010-030290.

A. R. Criado, C. de Dios and P. Acedo are with the Electronics Technology Department, Universidad Carlos III de Madrid, Leganés, Madrid 28911 Spain (e-mail: cdios@ing.uc3m.es). introduced considering elements as Mach-Zehnder modulators [4], electro-absorption modulators [5] or dual-mode monolithic laser sources [6]. These configurations have shown polarization dependence or low conversion efficiencies. These drawbacks have somehow hidden the advantages of these photonic schemes. Recently, Semiconductor Optical Amplifiers (SOA) have also been proposed as optoelectronic mixers [7$10]$.

Optical mixing using SOAs is usually accomplished in alloptical [9, 10] configurations, but also Electro-Optical (EO) schemes have been recently reported [7, 8]. In the EO approach, which is the most interesting in terms of obtaining a photonic heterodyne mixer, one of the electrical signals involved (i.e. the local oscillator (LO) or the radio-frequency $(\mathrm{RF})$ ) is modulated onto an optical carrier that is delivered to the optical input of the SOA. The other electrical signal is directly applied to the electrical port of the SOA modulating its bias voltage. Both down-conversion [8] and up-conversion [7] have been reported. These studies [7, 8] focus on the use of SOA-based EO mixing schemes for modulated RF signal distribution using Radio over Fiber (RoF) techniques. All the reported systems use polarization-dependent external modulators, and provide with sufficient mixing efficiency only in a limited range of frequencies [8].

In this work, we present the detailed study of a new scheme for electro-optical (EO) downconversion using a SOA where the LO and the retrieved IF signals are remotely distributed over fiber to/from a single COTS Ultra Non Linear SOA (XNSOA) that acts as a mixer device in the RF detection front-end. The photonic LO is generated modulating a DFB under Gain Switching (GS) operation and the RF electrical signal is directly applied to the mixer (SOA). This eliminates the requirement of other optical components (e.g. external modulators and polarization controllers) and the subsequent polarization dependency, thus having a dramatically improvement in complexity, size, cost, energy efficiency and compactness of the system. Downconversion ratios up to -5.65 $\mathrm{dB}$ have been obtained in a wide frequency range (from 2 to 15 $\mathrm{GHz}$ ), showing a broadband performance of the proposed heterodyne optoelectronic receiver. These results are specially promising for the development of scalable antenna arrays with photonic distribution of LO. 


\section{ELECTRO-OPTICAL MIXING SETUP}

The scheme introduced in this work is depicted in Fig. 1. The LO signal $\left(\mathrm{f}_{\mathrm{LO}}\right)$ is applied to a DFB diode laser (QPhotonics QDFBLD-1550-50) that works under GS conditions. This approach permits the modulation of the optical carrier without external modulators. The photonic LO produced has an optical frequency comb-like spectra [11] that is distributed to the heterodyne receiver using a fiber link (Fig.1a). The LO is then coupled to the optical input of the Ultra Nonlinear SOA (CIP SOA-XN-OEC-1550), where the EO mixing process takes place when the RF electrical signal $\left(f_{R F}\right)$ introduced in the electrical port of the XN-SOA modulates its biasing point. The XN-SOA device has a small signal gain of $20 \mathrm{~dB}$ at bias at $150 \mathrm{~mA}$ and a maximum saturated optical power of $13 \mathrm{dBm}$. The obtained IF appears modulating the optical carrier at the output of the XN-SOA (Fig. 1c) and is recovered at the end of the optical downlink using a high bandwidth photodiode ( $\mathrm{u} 2 \mathrm{t}$ XPDV2120R) followed by a low-pass filter. This scheme is cost effective and takes advantage of the additional non-linearities of the mixer and its polarization independence.

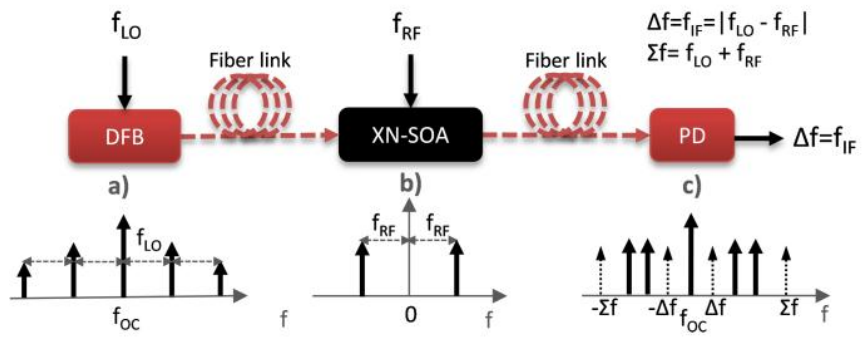

Fig. 1. EO mixing concept using an Ultra Non Linear SOA. (a) Optical output of GS DFB laser; (b) electrical signal modulating the XN-SOA bias; (c) optical output of the XN-SOA (only sum and difference terms are represented)

\section{EXPERIMENTAL RESULTS}

\section{A. Characterization of the optoelectronic devices}

The electronic frequency response of the transmission coefficients for the XN-SOA and the DFB are measured (Fig. 2). In both cases we obtain a $3-\mathrm{dB}$ bandwidth below $2 \mathrm{GHz}$. These preliminary results may lead to the wrong assumption that the proposed system will show an operational upper frequency limit in that range, but that it is not the case as it will be demonstrated later. In this sense, we have to keep in mind that we are using both DFB laser and XN-SOA in non-linear regimes (GS and saturation, respectively).

To illustrate this later point, in Fig. 2 (bottom) we show that for the optical input power values considered in our study $\left(\mathrm{P}_{\text {in }}>\right.$ $1.5 \mathrm{~mW}$ ) the transmission parameters of the $\mathrm{XN}-\mathrm{SOA}$ remain constant when its operating current is higher than $100 \mathrm{~mA}$. This demonstrate that the SOA is working under saturation condition; increasing its non-linear behavior and phenomena as cross-gain modulation (XGM) are enhanced [12]. These are the operating points of interest for the EO downconversion scheme.
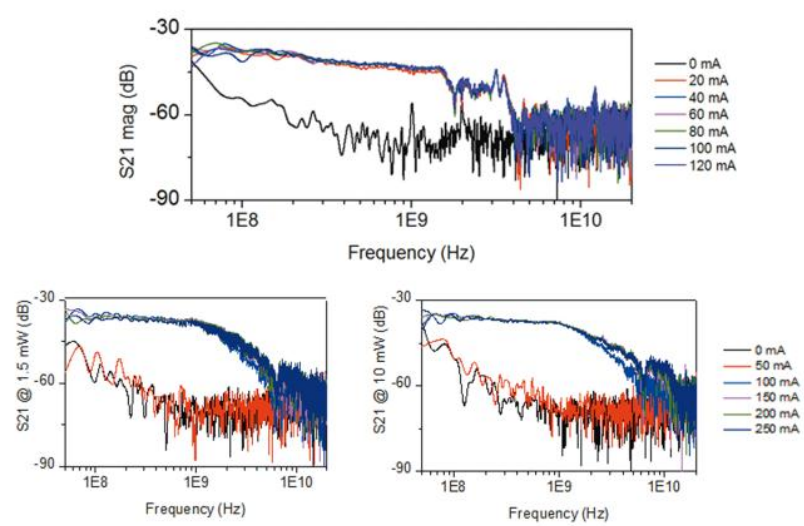

Fig. 2. Electro-optical transmission coefficients for GS DFB (top), and biasmodulated XN-SOA for $1.5 \mathrm{~mW}$ and $10 \mathrm{~mW}$ optical input power (bottom).

\section{B. Ultra-Non-Linear SOA performance as EO mixer}

The parameter we have used to evaluate the performance of the system has been the downconversion ratio, defined for optoelectronics mixers as the ratio between the electrical power of the downconverted signal at $\mathrm{f}_{\mathrm{IF}}$ (output of the photodiode) and the electrical power of the RF signal present after mixing at $f_{R F}$ [9]. The downconversion efficiency map for a wide frequency range is depicted in Fig 3 (top) for the system described in Fig.1. Also in Fig. 3 (bottom) the same map is shown for the case where the LO is applied to the electrical port of the SOA to allow a comparison with previously published results [8].
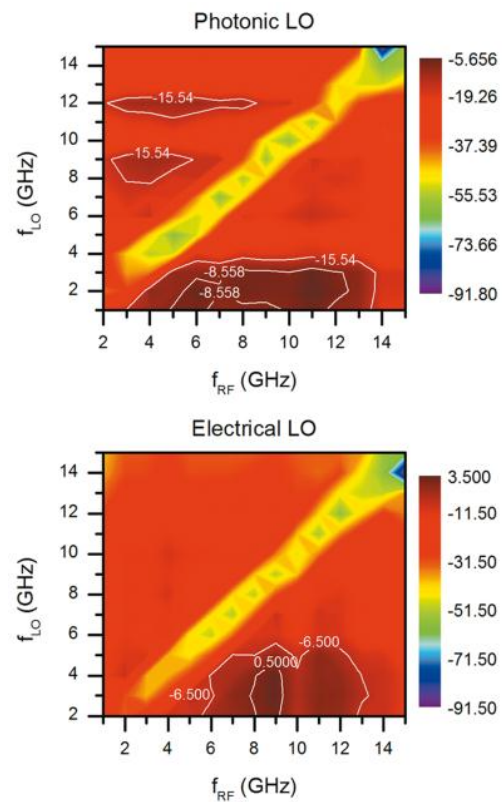

Fig. 3. Down-conversion ratio for the GS DFB as photonic LO (top) and for the bias modulated XN SOA as electrical LO (bottom).Contour lines represent $3 \mathrm{~dB}$ and $10 \mathrm{~dB}$ bandwidth.

An analysis of the frequency dependence of the downconversion ratio has been performed sweeping both $f_{\mathrm{LO}}$ and $\mathrm{f}_{\mathrm{RF}}$ up to $15 \mathrm{GHz}$. The bias current applied to the DFB is $40 \mathrm{~mA}$, for an average optical power around $5 \mathrm{~mW}$. The $\mathrm{XN}$ SOA is biased at $150 \mathrm{~mA}$ (saturation) and shows an average optical output power of $10 \mathrm{~mW}$. Regardless of the relatively high power introduced into the fiber, nonlinear effects in the 
fiber are not of relevance as the transmission lengths involved are small $(<500 \mathrm{~m})$.

The results shown in Fig.3 demonstrate some reciprocity in the behavior of the scheme. The maximum downconversion ratio when a photonic LO is used reaches $-5.65 \mathrm{~dB}$. This value is smaller than the one obtained when the LO is applied to the electrical port of the SOA $(3.5 \mathrm{~dB})$; however, the optimum downconversion area is flatter and wider with a photonic LO. These regions with better conversion ratios appear for RF frequencies in the $\sim 4$ to $13 \mathrm{GHz}$ range and $\mathrm{LO}$ frequencies below $4 \mathrm{GHz}$. The latter frequency limit for the LO is probably associated to the direct modulation bandwidth of the DFB under GS operation (Fig. 2, top). However, this limitation does not hold for the RF frequency as the system behaves properly even for frequencies much higher than the $3 \mathrm{~dB}$ bandwidth of the transmission coefficients for the SOA shown in section II (Fig. 2, bottom). This demonstrates the advantages of the use of a highly nonlinear SOA as presented in this work, since it can be used as an optoelectronic mixer well above its linear 3 $\mathrm{dB}$ modulation bandwidth showing broadband performance as a downconversion mixer. When both frequencies (LO and IF) are close to each other, the conversion ratio falls as expected (homodyne operation). Finally, and besides the demonstrated reciprocity of this system, we have observed also the possibility of using it for up-conversion [7]. This will be key to obtain a low cost, compact and polarization independent bidirectional system.

In order to complete the characterization of the optoelectronic mixer, in Fig. 4 we show the evolution of the downconversion ratio for a photonic LO scheme versus the $\mathrm{XN}-\mathrm{SOA}$ bias current at a given operation point $\left(\mathrm{f}_{\mathrm{LO}}=9.1 \mathrm{GHz}\right.$, $\mathrm{f}_{\mathrm{RF}}=8 \mathrm{GHz}$ ). It can be seen that for saturated operation of the $\mathrm{XN}-\mathrm{SOA}$ (above $100 \mathrm{~mA}$ ), the downconversion ratio presents an almost constant value. This is advantageous, since other optoelectronic mixing approaches do not show this stable performance under variations in the operation point of the SOA [8]. This confirms again the importance of operating the $\mathrm{XN}-\mathrm{SOA}$ in its high non-linear region to improve the mixing features of the device.

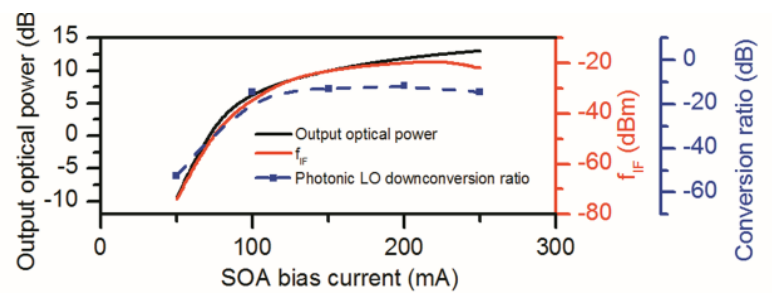

Fig. 4. Current dependence of the downconversion ratios (blue dashed), the average optical output power of the XN-SOA (solid black) and the power of the remotely recovered IF electrical signal (solid red).

\section{CONCLUSIONS}

A RF heterodyne receiver scheme based on electro-optical (EO) downconversion using a single COTS Ultra Non Linear SOA (XN-SOA) without the use of any external modulator has been presented. The performance of the system has been evaluated under remote fiber distribution of a photonic LO to the heterodyne receiver and optical retrieval of the IF signal. Downconversion has been experimentally observed in a wide range of frequencies showing a broadband operation. The dependence of the downconversion ratio on the bias current applied to the XN-SOA has also been studied, showing an almost constant value for saturated operation, what is an advantage over other mixing approaches that do not show this stability under variations in the operation point of the SOA. The proposed scheme presents reciprocity as it also behaves properly when the LO is electrically applied to the XN-SOA bias voltage.

This optoelectronic mixer could find applications in scalable antennas arrays to remotely process the received signal as the LO can be remotely delivered to the RF receiver (where the RF signal is directly applied to the SOA electrical port) and the IF retrieved using optical fiber. The final scheme will result in a very compact system, with low transmission losses, lightweight and EMI immunity, valid for a relatively wide frequency range (4 to $13 \mathrm{GHz}$ ).

\section{REFERENCES}

[1] A. J. Seeds and K. J. Williams, "Microwave Photonics," Lightwave Technology, Journal of, vol. 24, pp. 4628-4641, 2006

[2] J. Marti and J. Capmany, "Microwave photonics and radio-over-fiber research," Microwave Magazine, IEEE, vol. 10, pp. 96-105, 2009.

[3] N. Breuil, M. Dispenza, L. Morvan, A. M. Fiorello, S. Tonda, D. Dolfi, M. Varasi, and J. Chazelas, "New optical modulation schemes applied to local oscillator distribution in radar systems," in Microwave Photonics, 2004. MWP'04. 2004 IEEE International Topical Meeting on, 2004, pp. 119 122.

[4] T. Kuri, H. Toda, and K. Kitayama, "Dense wavelength-division multiplexing millimeter-wave-band radio-on-fiber signal transmission with photonic downconversion," Lightwave Technology, Journal of, vol. 21, pp. 1510-1517, 2003.

[5] B. Hraimel, Z. Xiupu, and W. Ke, "Photonic Down-Conversion of Millimeter Wave Multiband Orthogonal Frequency Division Multiplexing Ultra-Wideband Using Four Wave Mixing in an Electro-Absorption Modulator," Lightwave Technology, Journal of, vol. 28, pp. 1987-1993, 2010.

[6] P. Acedo, H. Lamela, and C. Roda, "Optoelectronic up-conversion using compact laterally mode-locked diode lasers," Photonics Technology Letters, IEEE, vol. 18, pp. 1888-1890, 2006.

[7] J. Palací, G. Villanueva, and J. Herrera, "EAM-SOA millimeter-wave frequency up-converter for radio-over-fiber applications," Optics Communications, vol. 284, pp. 98-102, 2011

[8] C. Bohémond, A. Sharaiha, T. Rampone, and H. Khaleghi, "Electrooptical radiofrequency mixer based on semiconductor optical amplifier," Electronics Letters, vol. 47, pp. 331-333, 2011.

[9] S. Jun-Hyuk, C. Chang-Soon, K. Young-Shik, C. Yong-Duck, K. Jeha, and C. Woo-Young, "SOA-EAM frequency up/down-converters for 60$\mathrm{GHz}$ bi-directional radio-on-fiber systems," Microwave Theory and Techniques, IEEE Transactions on, vol. 54, pp. 959-966, 2006.

[10] C. Bohémond, P. Morel, A. Sharaiha, T. Rampone, and B. Pucel, "Experimental and Simulation Analysis of the Third-Order Input Interception Point in an All-Optical RF Mixer Based on a Semiconductor Optical Amplifier," Lightwave Technology, Journal of, vol. 29, pp. 91-96, 2011.

[11] P. M. Anandarajah, R. Maher, Y. Q. Xu, S. Latkowski, J. O'Carroll, S. G. Murdoch, R. Phelan, J. O'Gorman, and L. P. Barry, "Generation of Coherent Multicarrier Signals by Gain Switching of Discrete Mode Lasers," Photonics Journal, IEEE, vol. 3, pp. 112-122, 2011.

[12] N. K. Dutta and Q. Wang, Semiconductor optical amplifiers. Singapore ; Hackensack, NJ: World Scientific Pub., 2006. 